\title{
TRASPLANTE RENAL EN ECUADOR, PUNTOS CLAVE Y SITUACIÓN ACTUAL
}

\section{Albán Tigre Jorge ${ }^{1}$, Villarreal Juris Andrea ${ }^{2 *}$, Mora Betancourt Juan ${ }^{3}$, Betancourt Nole Víctor ${ }^{4}$}

DOI: $10.48018 / \mathrm{rmv} . v 31 . i 2.6$

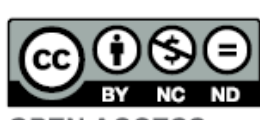

Este artículo está bajo una licencia de Creative Commons de tipo ReconocimienOPEN ACCESS derivadas 4.0 International.

1. Pontificia Universidad Católica del Ecuador. Facultad de Medicina. Posgrado de Cirugía General y Laparoscopía. Quito - Ecuador

2. Universidad Central del Ecuador. Facultad de Ciencias Médicas. Escuela de Medicina. Quito - Ecuador. 3. Universidad Técnica Particular de Loja. Área Biológica y Biomédica. Carrera Medicina. Loja - Ecuador. 4. Hospital de Especialidades FF.AA. Nro. 1. Médico del Servicio de Cirugía General y Trasplante. Quito - Ecuador.

ORCID ID:

Albán Tigre Jorge

orcid.org/0000-0002-0456-0561

Villarreal Juris Andrea

orcid.org/0000-0002-0977-3179

Mora Betancourt Juan

orcid.org/0000-0002-9836-830X

Betancourt Nole Víctor

orcid.org/0000-0002-0007-9447

*Corresponding author: Villarreal Juris Andrea E-mail: andrea.fima_95@hotmail.com

Article history

Received: 19 - Sep - 2020

Accepted: 03 - Nov - 2020

Publish: $01-$ Dic - 2020

STROBE 2008 Check List statement: The authors have real the STROBE 2008 Check List and the manuscript was prepared and revised according to the STROBE 2008 Checklist.

Conflict of interest: All authors declared that there are no conflicts of interest.

Financial disclosure: The authors have no financial relationships relevant to this article to disclose

Authors' contribution: All the authors reviewed and approved the final manuscript.

Forma de citar este artículo: Albán Tigre J, Villarreal Juris A, Mora Betancourt J, Betancourt Nole V. TRASPLANTE RENAL EN ECUADOR, PUNTOS CLAVE Y SITUACIÓN ACTUAL. Rev Med Vozandes. 2020; 31 (2): 42 - 48

\section{Resumen}

\section{Introducción}

El trasplante renal en Ecuador inició en 1976, fue limitado hasta 2011, donde entró en vigor la Ley Orgánica de Donación y Trasplante de Órganos, Tejidos y Células. Está indicado en insuficiencia renal terminal, para pacientes adultos y pediátricos; y, en comparación con diálisis peritoneal y renal, genera menor gasto para el sistema de salud. En 2015, en Ecuador, al menos 30000 personas padecían enfermedad renal crónica terminal; $45 \%$ de ellas podían fallecer sin tratamiento. El objetivo de este estudio fue determinar la situación actual de Ecuador respecto al trasplante renal.

\section{Metodología}

Estudio descriptivo, retrospectivo. Se revisó estadística del INDOT desde 2007 hasta agosto 2019, para determinar número total de trasplantes y trasplantes renales, tipo de trasplante, donantes efectivos, tasa de donantes por millón de habitantes (pmh), tasa de órganos trasplantados, evolución de la lista de espera nacional, tasa de supervivencia, etc.

\section{Resultados}

Desde 2007 hasta 2019 se realizaron 6134 trasplantes de órganos y tejidos: $25.4 \%$ renales. La mayoría de los donantes fue de sexo masculino (68.1\%). La tasa de donantes (pmh) entre 2009 a 2019 fue 4.2 (DS: \pm 2.1 ). La tasa media de órganos trasplantados fue 8.1 (DS: \pm 3.6 ) (pmh) desde 2007 hasta 2019. En este periodo se realizaron 1560 trasplantes renales: $83.1 \%$ con donante cadavérico ( $88.1 \%$ adultos; $11.9 \%$ pediátricos) y $16.9 \%$ con donante vivo ( $72.4 \%$ adultos; $27.6 \%$ pediátricos). La tasa de supervivencia al año tras trasplante renal cadavérico y con donante vivo fue 0.94 y 0.97 en adultos; y 0.90 y 0.97 en pediátricos, respectivamente.

Actualmente existen solo 5 centros acreditados para trasplante renal en adultos y uno para trasplante renal pediátrico

\section{Conclusiones}

El trasplante renal ha tenido un avance significativo en Ecuador, sin embargo, aún se encuentra por debajo de la meta establecida por la Organización Mundial de la Salud (OMS) para el manejo adecuado de los pacientes con falla renal crónica.

Palabras clave: Trasplante de Riñón, Trasplante, Trasplante de Órganos, Ecuador 


\section{Abstract \\ Keywords: Kidney Trans- plantation, Transplantation, Organ Transplantation, \\ KIDNEY TRANSPLANT IN ECUADOR, KEY POINTS AND CURRENT SITUATION.} Ecuador

\begin{abstract}
Introduction
Kidney transplantation in Ecuador began in 1976, it was limited until 2011, when the Organic Law of Donation and Transplantation of Organs, Tissues and Cells became valid. This is indicated in end-stage renal failure, in adult and pediatric patients; and, compared to peritoneal and renal dialysis, it is less expensive for the health system. In 2015, in Ecuador, at least 30,000 people suffered from end-stage kidney disease; $45 \%$ of them could die without treatment. The objective of this study was to determine the current situation in Ecuador regarding kidney transplantation.
\end{abstract}

\begin{abstract}
Methodology
A descriptive, retrospective study. INDOT statistics were reviewed from 2007 to August 2019, to determine the total number of transplants and kidney transplants, type of transplant, effective donors, rate of donors per million population (pmp), transplanted organ rate (pmp), evolution of the national waiting list, survival rate, etc.

Results

From 2007 to 2019, 6134 transplants were performed: $25.4 \%$ renal. Most donors were male (68.1\%). The donor rate (pmp) between 2009 and 2019 was 4.2 (SD: \pm 2.1 ). The mean rate of transplanted organs was 8.1 (SD: \pm 3.6 ) (pmp) from 2007 to 2019. In this period 1560 kidney transplants were performed: $83.1 \%$ with cadaveric donors $(88.1 \%$ adults; $11.9 \%$ pediatric) and $16.9 \%$ with living donors ( $72.4 \%$ adults; $27.6 \%$ pediatric). The one-year survival rate after cadaveric and living donor kidney transplantation was 0.94 and 0.97 in adults; and 0.90 and 0.97 in pediatrics, respectively. Currently there are only 5 accredited centers for kidney transplantation in adults and one for pediatric kidney transplantation .
\end{abstract}

\section{Conclusions}

Kidney transplantation has made significant progress in Ecuador, however, it is still below the World Health Organization (WHO) goal established for the proper management of patients with chronic kidney failure.

\section{Introducción}

Junto con la expectativa de vida, la enfermedad renal crónica ha ido en aumento a nivel mundial; desafortunadamente, no existe una estimación fiable del impacto que produce sobre el sistema de salud global, al encontrarse anexa a patologías como obesidad, hipertensión arterial, diabetes mellitus, entre otras. Involucra múltiples estratos sociales y llama la atención el escaso acceso a una atención médica de calidad y multidisciplinaria para su manejo, dependiente de varios factores, que incluyen demografía y políticas de salud (1).

El trasplante renal está indicado en insuficiencia renal terminal, tanto en pacientes adultos como pediátricos, independientemente de la causa (nefropatía diabética, enfermedad renal poliquística, glomerulonefritis crónica, etc.) ${ }^{(2,3)}$.

La prevalencia de enfermedad renal en el año 2014 alcanzó el 14.8\% en Estados Unidos, según el Sistema Nacional de Datos Renales (US Renal Data System- USRDS), de este porcentaje, el $3.5 \%$ (678383 pacientes) fue tratado con terapia de sustitución renal, superando el $20 \%$ de todo el gasto en salud de los mayores a 65 años (4). De acuerdo al registro de la Red Unida para el Intercambio de Órganos (United Network 
for Organ Sharing -UNOS), que se incorporó en este país en 1984 como una organización privada sin fines de lucro, que surgió de la Fundación de Adquisición de Órganos del Sureste (South-Eastern Organ Procurement Foundation) a fin de que los trasplantes tengan éxito y la calidad de vida mejore ${ }^{(5)}$, se llevaron a cabo 355812 trasplantes renales desde 1995 hasta 2012, con una tasa aproximada de supervivencia del injerto a 3 años del 79\% para los trasplantes entre 1998 a $2001^{(6,7)}$.

De manera similar, de acuerdo al registro de la Asociación Renal Europea - Asociación Europea de Diálisis y Trasplantes (European Renal Association - European Dialysis and Transplant Association: ERA-EDTA) que se estableció a mediados de la década de $1960{ }^{(8)}$, en 2015 y 2017, 546783 y 592779 pacientes recibieron terapia de sustitución renal por enfermedad renal terminal; y se realizaron 21056 y 22902 trasplantes renales, respectivamente ${ }^{(9,10)}$. Además, para los pacientes que comenzaron terapia de sustitución renal durante 2006-2010, la probabilidad de supervivencia no ajustada del paciente a 5 años fue de $50.0 \%$, mientras que durante $2008-2012$ fue de $50.8 \%{ }^{(9,10)}$.

En Colombia, incluyendo el programa de trasplante, la enfermedad renal crónica generó una carga económica al sistema de salud entre 8.7 y 14.4 billones COP, representando entre el $1.6 \%$ y el $2.7 \%$ del PBI en el $2015^{(11)}$.

En el Ecuador, con base en las directrices de la Sociedad Latinoamericana de Nefrología e Hipertensión (SLANH) y de acuerdo con la Tercera Encuesta de Salud y Nutrición (NANHES III), con 16278844 habitantes, se determinó que al menos 30000 personas padecían enfermedad renal crónica en estadío terminal, y $45 \%$ de ellas podía fallecer sin haber iniciado el tratamiento de sustitución renal. Según la indicación de diálisis peritoneal o renal, los pacientes generan un gasto para el sistema nacional de salud que oscila entre 1300 y 1456 dólares por mes, alcanzando 17472 dólares por año. Más del 70\% de los casos supera los cinco años, obteniendo un gasto total por paciente de aproximadamente 87360 dólares ${ }^{(4,11-13)}$.

El trasplante renal en Ecuador da inicio en 1976 con un donante vivo en el Hospital de Especialidades de las Fuerzas Armadas Nro. 1, sin embargo, la primera Ley de Trasplante de Órganos y Tejidos fue implementada en $1994{ }^{(14)}$, en ausencia de apoyo económico gubernamental, progresando de manera inconstante y limitada debido a las dificultades sociopolíticas, siendo hasta marzo de 2011, que tras la aprobación de la Ley Orgánica de Donación y Trasplante de Órganos, Tejidos y Células, evoluciona verdaderamente el programa de trasplante renal y se incorpora de forma protocolaria al sistema de salud pública del Ecuador, aunque aún con carencias ${ }^{(15,16)}$.

Actualmente el programa de trasplante renal es regulado por el Instituto de Donación y Trasplante de Órganos, Tejidos y Células (INDOT), de acuerdo a los principios de trasplante de la Organización Mundial de la Salud (OMS), cumpliendo, en teoría, estándares internacionales en cuanto a la selección, manejo y seguimiento de los pacientes y donantes ${ }^{(15)}$.

En este trabajo se pretende analizar los datos proporcionados por el INDOT a fin de establecer la situación actual de Ecuador con respecto al trasplante renal.

\section{Metodología}

Este estudio fue llevado a cabo de manera retrospectiva y descriptiva, siguiendo las directrices propuestas en las guías y el checklist de Strengthening The Reporting of Observational Studies in Epidemiology (STROBE) para estudios observacionales ${ }^{(17)}$ y fue aprobado pro el Comité de Ética del Hospital de las FFAA Nro 1.

Se revisaron los informes estadísticos de la base de datos nacional del INDOT a partir de 2007 hasta agosto 2019, para determinar el número total de trasplantes, número de trasplantes renales, tipo de trasplante, donantes efectivos, tasa de donantes por millón de habitantes (pmh), tasa de órganos trasplantados (pmh), evolución de la lista de espera nacional, tasa de supervivencia al mes, al año, a los cinco y diez años, datos demográficos. La tasa de supervivencia del injerto y análisis de costo beneficio no pudieron ser llevados a cabo debido a datos insuficientes.

El análisis se llevó a cabo mediante la herramienta JASP 0.11.1.0 respaldada académicamente por la Universidad de Ámsterdam. Las variables categóricas se reportaron como porcentajes, las variables continuas fueron expresadas como media y desviación estándar. Las tasas de donantes y de órganos trasplantados se reportaron por millón de habitantes.

\section{Resultados}

Los reportes del INDOT se encuentran incompletos a partir del año 2007 hasta 2010, en concordancia con la falta de implementación de un protocolo para trasplante renal sino hasta 2011, tras la vigencia de la nueva Ley Orgánica de Donación y Trasplantes de Órganos, Tejidos y Células y la creación de la lista de espera única nacional. A pesar de ello, los registros desde 2011 a 2012 y desde 2015 a 2019 carecen de información como causas de pérdida de donantes.

Desde 2007 hasta 2019 se realizaron 6134 trasplantes de órganos y tejidos, el $25.4 \%$ de ellos renales. La mayoría se realizaron dentro de la jurisdicción de la Zona 1 de salud (68.2\%), seguidos por la Zona 2 (17.2\%) y Zona 3 (14.6\%). Hasta 2019 el $91 \%$ de los ecuatorianos cedulados mantuvo su condición de donante.

En 2013 y 2014 se reportó 110 y 115 casos de muerte encefálica, sin embargo, los donantes efectivos fueron apenas 63 y 50 , respectivamente; las causas para pérdida de donantes englobaron oposición familiar a la donación (61.6\%), contraindicación médica para la donación (26.8\%), voluntad contraria al trasplante manifestada en el registro 
civil (10.7\%), e ilegalidad del donante en el país o ausencia de familiares (0.9\%). En 2014, con respecto a la pérdida de riñones, adicionalmente a las anteriores, se reportó falta de receptor compatible (5\%), riñón poliquístico (15\%), esclerosis renal (10\%), hematoma retroperitoneal (5\%), pérdida de la cadena de frío $(0.9 \%)$, tiempo de isquemia prolongada (3.5\%) y trasplante en bloque $(0.9 \%)$.

Tabla 1. Ingresos a LEUN renal, tasas de donantes y de órganos trasplantados por año

$\begin{array}{llll}\text { Año } & \begin{array}{l}\text { Ingresos a } \\ \text { LEUN renal }\end{array} & \begin{array}{l}\text { Tasa de donantes } \\ \text { (pmh) }\end{array} & \begin{array}{l}\text { Tasa de órga- } \\ \text { nos trasplan- } \\ \text { tados (pmh) }\end{array} \\ 2007 & \text { SD } & \text { SD } & 4.15 \\ 2008 & \text { SD } & \text { SD } & 3.95 \\ 2009 & \text { SD } & 1.22 & 4.07 \\ 2010 & \text { SD } & 2.34 & 6.53 \\ 2011 & 300 & 2.14 & 6.48 \\ 2012 & \text { SD } & 3.48 & 8.25 \\ 2013 & 246 & 3.99 & 9.83 \\ 2014 & 349 & 3.12 & 7.86 \\ 2015 & 445 & 3.9 & 8.23 \\ 2016 & \text { SD } & 5.1 & 10.65 \\ 2017 & 214 & 5.05 & 11.38 \\ 2018 & 420 & 7.76 & 16.33 \\ 2019 & 289 & 7.75 & 16.32 \\ \text { *SD: sin dato. } & & \\ \text { PMH, por millón de habitantes; LEUN, lista de espera única } \\ \text { nacional }\end{array}$

Desde 2011 hasta 2019, la edad media de los donantes fue 35.6 años (DS: \pm 3.2 ), la mayoría fue de sexo masculino (68.1\%; [511/750]) vs femenino (31.9\%; [239/750]); y las causas para muerte encefálica englobaron trauma craneoencefálico
(TCE) $(64.1 \%)$, accidente cerebrovascular (ACV) $(28.8 \%)$ y anoxia cerebral $(7.1 \%)$.

En el apartado de trasplante renal de la lista de espera única nacional hubo un ingreso promedio de 320.3 (DS: \pm 80.1 ) personas entre 2011 a 2019 (Tabla 1). Esta lista es dinámica y está muy lejos de ser concordante entre oferta y demanda; variando los pacientes activos entre 159 en 2011, 284 en 2013, hasta 73 en 2018 y 121 en 2019; con una media de 180 (DS: \pm 86.5 ). Lamentablemente, el INDOT no ofrece datos acerca de la mortalidad en lista de espera ni las causas de abandono de esta.

Entre 2009 a 2019, se obtuvo una tasa media de 4.2 (DS: \pm 2.1 ) donantes por millón de habitantes (pmh), que no logra alcanzar el objetivo promedio determinado en el país de 6 (pmh). Desde 2007 hasta 2019, la tasa media de órganos trasplantados fue 8.1 (DS: \pm 3.6 ) (pmh) (Tabla 1).

Se realizó un total de 1560 trasplantes renales en este periodo de tiempo, de los que el $83.1 \%$ correspondieron a trasplantes con donante cadavérico y el $16.9 \%$ restante a trasplantes con donante vivo (Gráfico 1). El $88.1 \%$ de los primeros se realizó en adultos y el $11.9 \%$ en pacientes pediátricos; mientras que el $72.4 \%$ de trasplantes con donante vivo se realizó en pacientes adultos y el $27.6 \%$ en pediátricos. La edad media de los donantes cadavéricos fue 39 años (DS: \pm 1.7 ), mientras que la de donantes vivos fue 32.7 años (DS: \pm 7.2 ).

La tasa media de supervivencia tras trasplante renal cadavérico en pacientes adultos fue 0.98 (98\%) dentro del primer mes desde la recepción del órgano, 0.94 (94\%) al año, 0.85 (85\%) a los 5 años y 0.80 (80\%) a los 10 años. Mientras que la

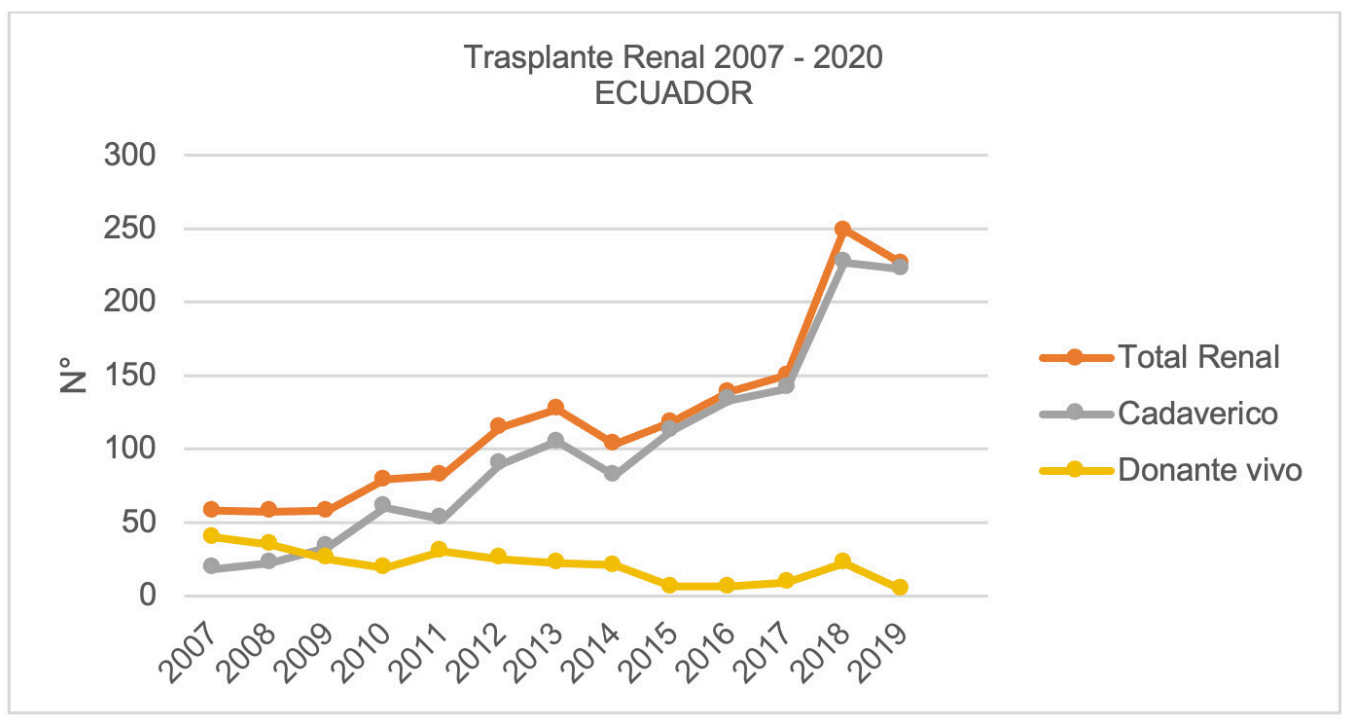

Gráfico 1. Evolución del trasplante renal en Ecuador Fuente: Elaborado por los autores 


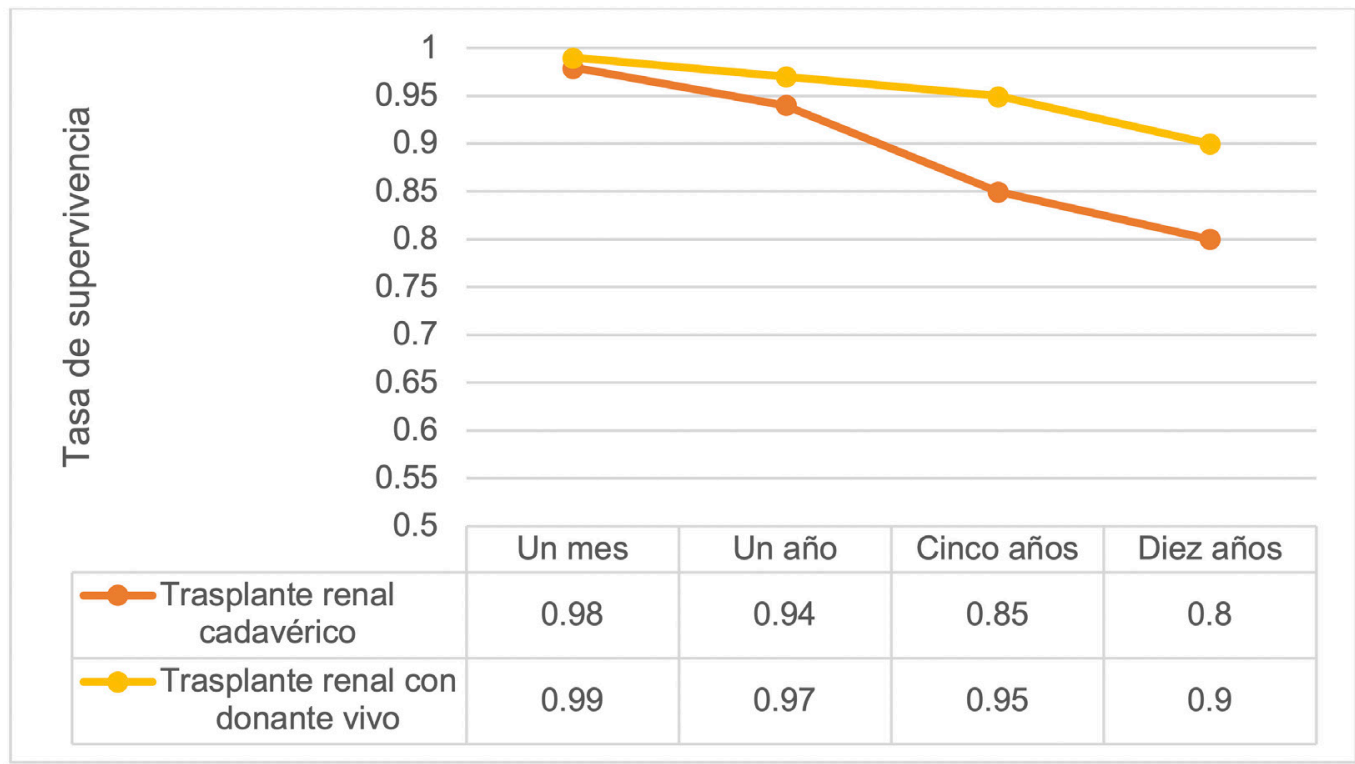

Gráfico 2. Supervivencia para trasplante

Fuente: Elaborado por los autores renal en pacientes adultos

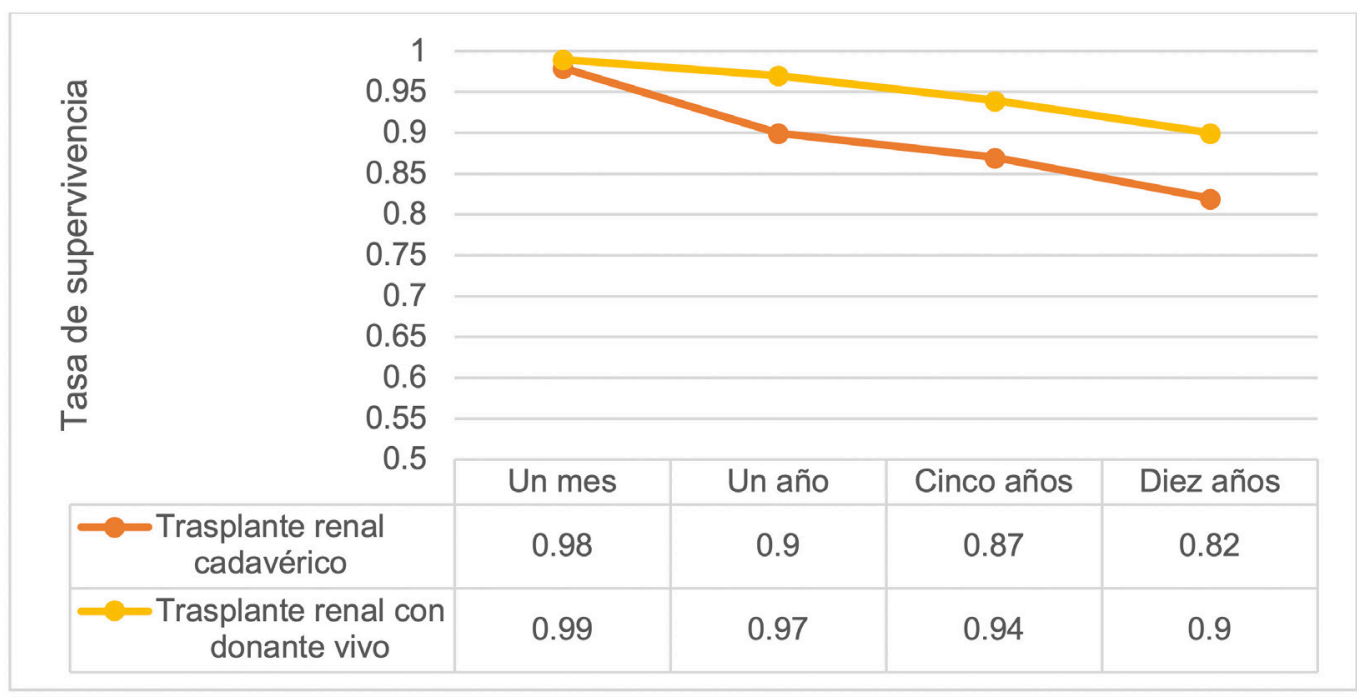

Gráfico 3. Supervivencia para trasplante renal en pacientes pediátricos

Fuente: Elaborado por los autores

tasa media de supervivencia tras trasplante renal con donante vivo fue 0.99 (99\%) dentro del primer mes, 0.97 (97\%) al primer año, 0.95 (95\%) a los 5 años y 0.90 (90\%) a los 10 años (Gráfico 2).

La tasa de supervivencia media posterior a trasplante renal cadavérico en pacientes pediátricos fue 0.98 (98\%) al mes, 0.90 (90\%) al año, 0.87 (87\%) a los 5 años y 0.82 (82\%) a los 10 años; y posterior a trasplante renal con donante vivo, alcanzó 0.99 (99\%) al mes, 0.97 (97\%) al año, 0.94 (94\%) a los 5 años y 0.90 (90\%) a los 10 años (Gráfico 3).

Hasta 2017 existieron once centros acreditados por el INDOT para trasplante renal cadavérico y con donante vivo en adultos: tres de ellos en Guayaquil, seis en Quito y dos en Cuenca; y un centro acreditado para trasplante renal pediátrico cadavérico y con donante vivo en Quito (Hospital Pediátrico Baca Ortiz).

Los promedios anuales de trasplantes renales variaron en cada institución: Hospital de Especialidades Eugenio Espejo realizó un promedio de 13 trasplantes renales; Hospital Carlos Andrade Marín un promedio de 18; Hospital de las Fuerzas Armadas $\mathrm{N}^{0} 1$ un promedio de 6 trasplantes renales; Hospital Metropolitano un promedio de 15; Hospital de la Policía en promedio 2 trasplantes; Hospital de los Valles en promedio 2 trasplantes; Hospital Teodoro Maldonado Carbo realiza en promedio 3 trasplantes al año; Hospital Luis Vernaza un 
promedio de 14 trasplantes; Clínica Kennedy en promedio 1 trasplante renal anual; Hospital Monte Sinaí un promedio de 2 trasplantes y el Hospital José Carrasco Arteaga realiza en promedio 6.

Actualmente existen solo 5 centros acreditados para trasplante renal en adultos según lo reportado en SINIDOT (Sistema Nacional Informático de Donación y Trasplante- implementado a partir de 2016): dos en Quito (Hospital Eugenio Espejo: con donante cadavérico y vivo, Hospital de los Valles: con donante vivo), dos en Guayaquil (Hospital Teodoro Maldonado Carbo, Hospital Luis Vernaza: ambos con donante cadavérico y vivo) y uno en Cuenca (Hospital José Carrasco Arteaga: con donante cadavérico y vivo); y un centro acreditado para trasplante renal pediátrico con donante cadavérico y vivo en Quito (Hospital Pediátrico Baca Ortiz).

\section{Discusión}

El trasplante como terapéutica en la enfermedad renal crónica está limitado a la disponibilidad de órganos, recursos médicos y tecnológicos. En 2014, la lista de espera en Estados Unidos alcanzó 88231 individuos, y con tan solo 18000 trasplantes se alcanzó al 20.4\% de la población objetivo; en Ecuador durante el mismo año la lista de espera alcanzó 349 individuos y se realizaron 103 trasplantes, correspondiendo al $29.5 \%$ de la población objetivo, sin embargo, según estimaciones internacionales, en Ecuador para el año 2015, a partir de la prevalencia de enfermedad renal crónica, debieron realizarse 1146 trasplantes en comparación con los 118 (10.3\%) reportados, demostrando el infra diagnóstico de enfermedad renal crónica en el país ${ }^{(4)}$.

A pesar de que el trasplante renal se percibe como la opción más rentable para el tratamiento de la enfermedad renal crónica, fallas en la cadena de procesamiento de órganos - complicaciones como la falla del injerto, incrementan notoriamente el costo del trasplante renal, es así como para pacientes con alto riesgo de complicaciones posoperatorias la terapia de sustitución renal puede tener mayor beneficio.

Para considerar como efectiva la opción del trasplante renal, este deberá durar al menos 5 años para no sobrepasar el gasto de un paciente en terapia de sustitución renal, que alcanza 87360 dólares en el mismo periodo de tiempo ${ }^{(13,18)}$. Por ejemplo, en países como Brasil, en 4 años, el trasplante renal cadavérico generó un ahorro por paciente de $R \$ 37$ mil y $R \$ 74$ mil en relación a hemodiálisis y diálisis peritoneal, respectivamente: y el trasplante renal con donante vivo, un ahorro de $R \$ 46$ mil y $R \$ 82$ mil, en relación a hemodiálisis y diálisis peritoneal, respectivamente ${ }^{(19,20)}$. Los desbalances económicos podrían explicar por qué no se ha incentivado la creación de centros de trasplantes en Ecuador, sin embargo, se requieren análisis de costo beneficio que abarquen todo el territorio nacional para comprobar esta hipótesis.

Como demuestran los reportes de INDOT, del total de muertes encefálicas reportadas entre 2013 y 2014, para el programa de trasplantes solo se captó al $52 \%$ de individuos debido a oposición familiar a la donación, voluntad contraria al trasplante manifestada en el registro civil, ilegalidad del donante en el país, entre otros. En 2009, la tasa de donantes en Ecuador fue de 1.2 (pmh), sin embargo, decisiones políticas oportunas permitieron elevar la misma hasta 7.76 (pmh) en 2018, superando la tasa de 6.3 (pmh) en América Latina, pero no equiparable a la de 31.7 (pmh) en Estados Unidos en el mismo año ${ }^{(21,22) .}$

Cabe recalcar las limitaciones de este estudio, entre las que se incluye su carácter retrospectivo y la ausencia de datos completos a lo largo de los años, que han impedido el cálculo de indicadores como supervivencia del injerto y realizar análisis de costo beneficio. En Colombia, por ejemplo, con respecto a trasplantes renales realizados entre 2008 a 2012, la supervivencia al año del injerto fue de $97.2 \%$, a los tres años fue de $93.2 \%$, y a los cinco años de $90,8 \%{ }^{(23)}$. De manera similar en 2015 a 5 años fue de $88.4 \%$ (24). En México, desde 2003 a 2009, la supervivencia del injerto fue de $83.9 \%$ y $94.3 \%$ a los 12 meses para trasplantes con donante cadavérico y donante vivo, respectivamente ${ }^{(25)}$. En Chile, la supervivencia del injerto renal en el periodo 1994 a 2003 fue de $87 \%$ al año, $82 \%$ a los 3 años, $79 \%$ a los 5 años y 59\% a los 10 años; y de 2004 a 2012 alcanzó $83 \%$ al año, $77 \%$ a los 3 años, $68 \%$ a los 5 años y $64 \%$ a los 10 años ${ }^{(26)}$.

\section{Conclusiones}

El trasplante renal ha tenido un avance significativo en Ecuador, sin embargo, aún se encuentra por debajo de la meta establecida por la Organización Mundial de la Salud (OMS) para el manejo adecuado de los pacientes con falla renal crónica; este puede dar una nueva esperanza de vida a pacientes que padecen esta enfermedad y a pesar de la dificultad operativa asociada a un elevado costo, el beneficio en la calidad de vida supera estas limitaciones siempre y cuando se realice un seguimiento adecuado. La creación de políticas públicas para optimizar el manejo de datos podría garantizar análisis exactos y útiles para el progreso del trasplante renal en Ecuador. El camino aún es largo y se deberá comprometer a las autoridades competentes para alcanzar a toda la población objetivo. 


\section{Referencias}

1. Luyckx VA, Tonelli M. Stanifer JW. The global burden of kidney disease and the sustainable development goals. Bull World Health Organ. 1 de junio de 2018;96(6):414-422D.

2. Daga Ruiz D, Fernández Aguirre C, Segura González F, Carballo Ruiz M. Indicaciones y resultados a largo plazo de los trasplantes de órganos sólidos. Calidad de vida en pacientes trasplantados. Med Intensiva. 1 de agosto de 2008;32(6):296-303.

3. Martínez Urrutia M. Trasplante renal en el niño. Un trasplante renal de por vida. Cir Pediatr. 2020;33(3):103-9.

4. Saran R, Robinson B, Abbott KC, Agodoa LYC, Albertus P, Ayanian J, et al. US Renal Data System 2016 Annual Data Report: Epidemiology of Kidney Disease in the United States. American Journal of Kidney Diseases. 1 de marzo de 2017;69(3):A7-8.

5. Technology I of M (US) C on HC, Goodman C. United Network for Organ Sharing [Internet]. Medical Technology Assessment Directory: A Pilot Reference To Organizations, rectory: A Pilot Reference To Organizations,
Assessments, and Information Resources. Assessments, and Information Resources.
National Academies Press (US); 1988 [citado 24 de septiembre de 2020]. Disponible en: https://www.ncbi.nlm.nih.gov/books/ NBK218485/

6. Yacoub R, Nadkarni GN, Cravedi P, He JC Delaney VB, Kent R, et al. Analysis of OPTN/ UNOS registry suggests the number of HLA matches and not mismatches is a stronger independent predictor of kidney transplant survival. Kidney Int. 2018;93(2):482-90.

7. Cecka JM. The UNOS Renal Transplant Registry. Clin Transpl. 2002;1-20.

8. Mehls O, Donckerwolcke RAM, Ehrich JHH. Re-establishment of the ERA-EDTA Registry. Pediatr Nephrol. febrero de 2004;19(2):128-9

9. Kramer A, Pippias M, Noordzij M, Stel VS, Afentakis N, Ambühl PM, et al. The European Renal Association - European Dialysis and Transplant Association (ERA-EDTA) Registry Annual plant Association (ERA-EDTA) Registry Annual Report 2015: a summary.
ro de 2018;1 1 (1):108-22.

10. Kramer A, Boenink R, Noordzij M, Bosdriesz JR, Stel VS, Beltrán P, et al. The ERA-EDTA Registry Annual Report 2017: a summary. Clin Kidney J. 22 de junio de 2020;13(4):693-709.
11. Sarmiento-Bejarano H, Ramírez-Ramírez C, Carrasquilla-Sotomayor $M$, Alvis-Zakzuk NJ. Alvis-Guzmán N, Sarmiento-Bejarano H, et al. Economic burden of Chonic Kidney Disease in Colombia, 2015-2016. Revista Salud Uninorte. abril de 2019;35(1):84-100.

12. Maza J. Prevalencia de hiperparatiroidismo secundario a enfermedad renal crónica estadio cinco en pacientes bajo tratamiento de dio cinco en pacientes bajo tratamiento de
hemodiálisis en el centro de hemodiálisis VYR durante el 2017 [Internet]. [Quito - Ecuador]: durante el 2017 [Internet]. [Quito - Ecuador]: 2018. Disponible en: http://repositorio.puce. edu.ec/handle/22000/15398? show=full

13. Ministerio de Salud Pública del Ecuador. Prevención, diagnóstico y tratamiento de la enfermedad renal crónica. guía de práctica clínica. [Internet]. Quito - Ecuador: Dirección Clínica. [Internet]. Quito - Ecuador: Dirección
Nacional de Normatización; 2018. Disponible Nacional de Normatizaciót
en: http://salud.gob.ec

14. Ministerio de Salud Pública del Ecuador- Organismo Nacional de Trasplantes de Órganos y Tejidos. Ley Nacional de Trasplante de Órganos y Tejidos. Registro Oficial No $N^{\circ} 492$, Organos y Tejid
No58 jul 27, 1994.

5. Huertas J, Garrido D, Serpa F. Organ Transplantation in Ecuador: Transplantation. marzo de 2020;104(3):445-7.

6. Registro oficial N. 381. Ley Orgánica de Donación y Trasplante de Órganos, Tejidos y Células. Ecuador; 2011.

7. von Elm E, Altman DG, Egger M, Pocock SJ, Gøtzsche PC, Vandenbroucke JP, et al. The Strengthening the Reporting of Observational Studies in Epidemiology (STROBE) statement: guidelines for reporting observational studies. $\mathrm{J}$ Clin Epidemiol. abril de 2008;61 (4):344-9.

18. Evans RW, Kitzmann DJ. An economic analysis of kidney transplantation. Surgical Clinics. 1 de febrero de 1998;78(1):149-74.

19. Silva SB, Caulliraux HM, Araújo CAS, Rocha E. Una comparación de los costes del trasplante renal en relación con las diálisis en Brasil. Cadernos de Saúde Pública [Internet]. 2016 [citado 1 de octubre de 2020];32(6). 2016 [citado 1 de octubre de 2020];32(6). Disponible en: http://www.scielo.br/scielo.
php?script=sci abstract\&pid=s0102-31 1 X201 php? script=sci_abstract\&pid $=$ S0 102-31
$6000605005 \& \operatorname{lng}=$ en \&nrm=iso \&tlng $=$ pt
20. Lugon JR, Strogoff-de-Matos JP, Lugon JR, Strogoff-de-Matos JP. Comparing costs of renal replacement therapy in a Brazilian city: a case for improvement of our health policies. Brazilian Journal of Nephrology. junio de 2017:39(2):106-7.

21. Edicion Medica. Ecuador inicia «cruzada nacionalı que promueve mantener la condición de donantes de órganos, tejidos y células [Internet]. 2019 [citado 30 de junio de 2020]. Disponible en: https://www edicionmedica.ec/secciones/salud-pub ublica/ ecuador-inicia-cruzada-nacional-para-promover-la-importancia-de-mantener-la-condicion-de-donante-de-organos-94842

22. Ramos M. OPS/OMS Ecuador - "Hospital amigo de la donación", una iniciativa de Ecuador para fortalecer la procuración de órganos y tejidos I OPS/OMS [Internet]. Pan órganos y tejidos I OPS/OMS [Internet]. Pan American Health Organization / World Health Organization. 2019 [citado 30 de junio de 2020]. Disponible en: https://www.paho. org/ecu/index.php? option=com_content\& view $=$ article \&id $=2301$ : hospital-amigo-de-ladonacion-una-iniciativa-de-ecuador-parafortalecer-la-procuracion-de-organos-ytejidos\&ltemid $=360$

23. Osorio-Arango $K$, Beltrán-Durán $M$, AriasMurillo Y, Prieto F, Robayo A. Vista de Supervivencia de receptores de trasplante renal en Colombia, 2008-2012. Biomédica. 2017;37:175-83

24. Dávila FA, Luna RD, Rey DAP. Análisis de supervivencia del injerto post-trasplante renal. Revista Colombiana de Nefrología. 2015:2(2):96-101.

25. Ticona-Garrón AB, Álvarez-Rangel LE, Jiménez-Domínguez A, Cruz-Santiago J, MedinaUicab C, Meza-Jiménez G, et al. Supervivencia del paciente e injerto renal al año de trasplante de donante fallecido; comparación con resultados de donante vivo. Rev Mex Urol. 1 de noviembre de 2010;70(6):34753.

26. Rodríguez Vidal M, Parra Morales $M$, Rocha Ortiz S, Castro Salas M, Villalobos Sepúlveda C. Supervivencia del injerto y del receptor en el trasplante ren en el trasplante renal en el hospital Guillermo Gran Benavente (Concepción, Chie). Enfermería Nefrológica. diciembre de $2014 ; 17(4): 292-8$ 